\title{
What causes clumps in winds from Red Supergiants and Asymptotic Giant Branch stars?
}

\section{A. M. S. Richards ${ }^{* a}$ K. Assaf ${ }^{b a}$ A. Baudry ${ }^{c}$ R.J. Davis ${ }^{a}$ L. Decin $^{d}$ M. Elitzur ${ }^{e}$ S. Etoka ${ }^{f a}$ S.T. Garrington ${ }^{a}$ M.D. Gray ${ }^{a}$ E.E. Lekht ${ }^{g}$ J.J. Lim $^{h i}$ I. McDonald ${ }^{a}$ E. Mendoza ${ }^{j}$ K. Murakawa ${ }^{k}$ G. Rudnitskij ${ }^{g}$ H.J. van Langevelde ${ }^{l m}$ M. Wittkowski ${ }^{n}$ and J.A. Yates ${ }^{o}$}

${ }^{a} J B C A$, University of Manchester, UK. ${ }^{b}$ Dept. of Physics, University of Wasit, Iraq.

${ }^{c} L A B$, Univ. Bordeaux, France

${ }^{d}$ Instituut voor Sterrenkunde, Katholieke Universiteit Leuven, Belgium.

${ }^{e}$ Dept. of Physics and Astronomy, University of Kentucky, USA.

${ }^{f}$ Hamburger Sternwarte, Germany. ${ }^{g}$ SAI, Lomonosov Moscow State University, Russia.

${ }^{h}$ Department of Physics, University of Hong Kong. ${ }^{i}$ ASIAA, Taipei, Taiwan.

${ }^{j}$ INAOE, Puebla, Mexico. ${ }^{k}$ School of Physics and Astronomy, University of Leeds, UK.

${ }^{l}$ JIVE, Dwingeloo, The Netherlands. ${ }^{m}$ Sterrewacht Leiden, Leiden University, The Netherlands.

${ }^{n}$ ESO, Garching, Germany. ${ }^{o}$ Dept. Physics and Astronomy, UCL, London, UK.

E-mail: amsrejb.man.ac.uk

Cool, evolved stars undergo copious mass loss but the details of how the matter is returned to the ISM is under debate. We investigated the structure and evolution of the wind at 5-50 stellar radii from Asymptotic Giant Branch and Red Super Giant stars using multi-epoch imaging of $\mathrm{H}_{2} \mathrm{O}$ and OH masers with MERLIN and VLBI, complemented by Pushchino and Nançay monitoring. The 22-GHz emission is concentrated in clumps which survive for decades although individual masers wink. In at least 5 sources, most matched features brighten or dim in concert within a few months-years throughout shells tens or hundreds of au across, suggesting that radiative processes dominate variability. One cloud in W Hya was caught passing in front of a background cloud, leading to 50 -fold, transient amplification. The $22-\mathrm{GHz}$ clouds, $>20 \times$ denser than the wind average, contain a substantial fraction of the mass loss, with a filling factor $<1 \%$. Cloud size is proportional to parent star size, with RSG clouds an order of magnitude bigger than those around AGB stars. This suggests that their properties are determined directly by the star and matter is ejected in clumps of $\sim 10 \%$ stellar radius, similar to the scale of convection cell models.

We imaged 6-GHz radio continuum from Betelgeuse, using e-MERLIN. The $\sim 230$-mas disc (radius $5 \times$ the optical photospheric radius) has brightness temperature $\approx 1250 \mathrm{~K}$, similar to previous VLA observations. However, we resolved two spots hotter than the maximum average photospheric temperature, possibly caused by chromospheric patches and/or pulsation/convection. We are planning a programme of multi-epoch, multi-frequency monitoring using ALMA, the EVLA and e-MERLIN to resolve a sample of evolved stars and reveal matter transport in the photosphere. $\mathrm{SiO}$ maser imaging will relate this to mass loss from the stellar surface whilst comparing ALMA, MERLIN and VLBI, dust, $\mathrm{H}_{2} \mathrm{O}$ and $\mathrm{OH}$ images will confirm or challenge the picture of dense, dusty clumps embedded in diffuse gas throughout the circumstellar envelope.

11th European VLBI Network Symposium \& Users Meeting,

October 9-12, 2012

Bordeaux, France 


\section{Mass loss from evolved stars}

Asymptotic Giant Branch (AGB) and Red Supergiant (RSG) stars have masses below and above $\sim 8 \mathrm{M}_{\odot}$, respectively. Typical optical photospheric radii $R_{\star}$ are 1 AU for AGB stars, 8 AU for RSG, with pulsational periods from $\sim 6$ months to a few years. They lose mass at $10^{-7}$ to $>10^{-5}$ $\mathrm{M}_{\odot} \mathrm{yr}^{-1}$, the more massive stars having the higher mass loss rates. The coolest stars $\left(T_{\text {eff }} 2500-\right.$ $3500 \mathrm{~K}$ ) show copious dust formation at $5 R_{\star}$ and have strong molecular lines, including masers. Oxygen-rich stars have $\mathrm{SiO}$ masers at 2-4 $R_{\star}, 22-\mathrm{GHz} \mathrm{H}_{2} \mathrm{O}$ masers at 5-50 $R_{\star}, \mathrm{OH}$ mainline $(1665 / 7 \mathrm{MHz})$ masers at $>20 R_{\star}$ and $\mathrm{OH} 1612-\mathrm{MHz}$ masers usually at hundreds $R_{\star}$, if present. We monitored a dozen objects (without known companions) at 22 and $1.6 \mathrm{GHz}$, using MERLIN ${ }^{1}$ and the EVN/global VLBI to provide 10-20 mas resolution at all frequencies, at various intervals over almost 10 yr. This complements more intensive SiO monitoring using the VLBA (e.g. [1]).

Mass loss from cool, evolved stars is thought to be initiated by pulsations, followed by radiation pressure on dust accelerating the wind to escape velocity [2]. Dust nucleation is likely to start at 2-4 $R_{\star}$ [22], in the region where $\mathrm{SiO}$ masers show both outflow and infall. Woitke ([23]), however, cast doubt on the ability of the sort of very small grains seen around O-rich stars to drive the wind. Thick dust shells are seen with inner radii around $5 R_{\star}$ (e.g. [6]) and, from there outwards, $\mathrm{H}_{2} \mathrm{O}$ and $\mathrm{OH}$ masers show steady acceleration ([3], [20]). So, how does the wind as far as the point where most dust has formed? This is a greater problem for younger RSG such as Betelgeuse, which already has a mass loss rate $\sim 10^{-6} \mathrm{M}_{\odot} \mathrm{yr}^{-1}$ [15] but does not fully form dust until $>20 R_{\star}$.

Stellar convection cells and magnetic effects could contribute to the levitation of material. Star spots on several RSG have been resolved by optical/IR interferometry and compared with convective models, e.g. VX Sgr [4]. These possibilities are supported by the clumpiness of the winds and by maser magnetic field measurements. We highlight results for 22- $\mathrm{GHz} \mathrm{H}_{2} \mathrm{O}$ masers in Section 2, and for Betelgeuse in Section 3, summarising current and further work in Section 4.

\section{Tracing mass loss with 22-GHz water masers}

MERLIN provided 10-20 mas resolution at $22 \mathrm{GHz}$, in $0.1-0.4 \mathrm{~km} \mathrm{~s}^{-1}$ velocity channels. Individual maser spots are resolved by fitting 2D Gaussian components with (sub-)mas accuracy, depending on the signal-to-noise ratio. Spatially close components in successive channels form series, often with (1D) Gaussian line shapes. These represent emission from discrete features, see Fig. 1, left. Some spatial features can be matched at epochs separated by up to 18 months for AGB stars and at least $5 \mathrm{yr}$ for RSG ([24], [11], [20] and references therein).

The proper motions of matched features show accelerating expansion consistent with the Doppler velocities, with no significant rotation. We fitted point-symmetric spheroidal shells, placing the star at the centre of expansion. Fig. 1, right, shows the angular separation from this centre as a function of velocity with respect to the Local Standard of Rest $\left(V_{\mathrm{LSR}}\right)$. This gives maser shell crossing times from about a decade to a century, for the smallest AGB stars to the largest RSG. The expansion velocity approximately doubles, passing through escape velocity. Comparing Fig. 1 with single-dish spectra (Fig. 2, left), shows that some spectral peaks, unambiguously identified

\footnotetext{
* Speaker.

${ }^{1}$ now e-MERLIN, the UK radio interferometer operated by the University of Manchester on behalf of STFC
} 

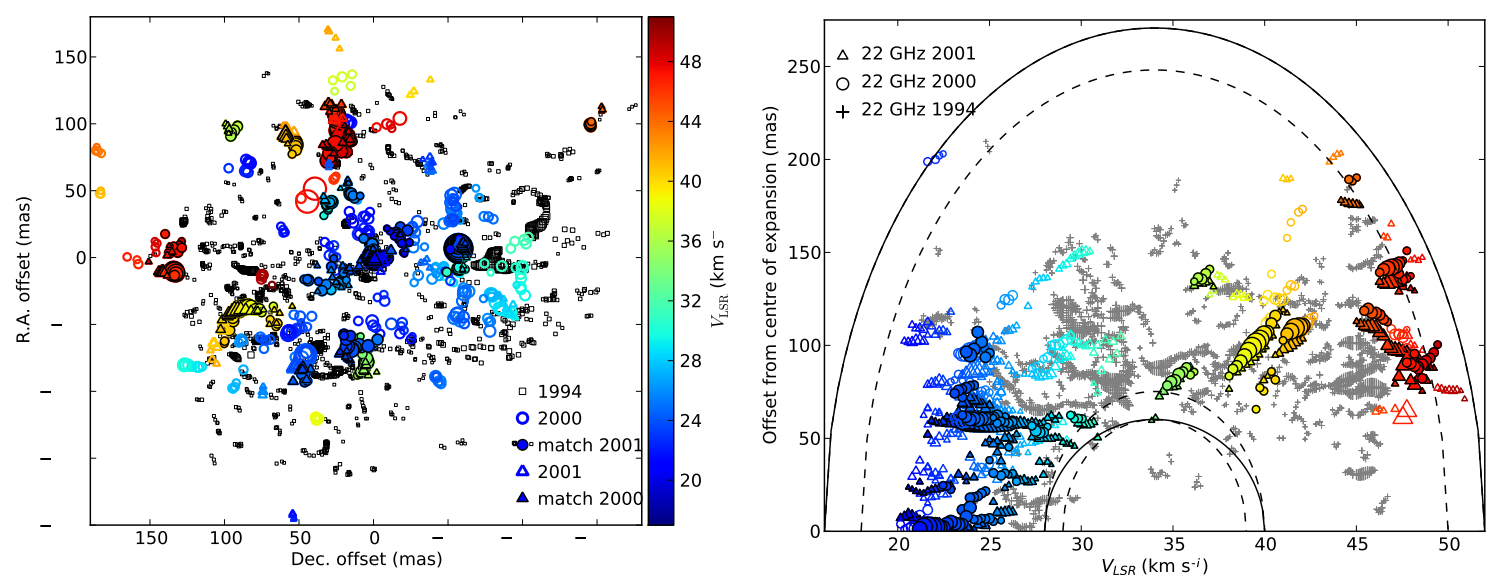

Figure 1: 22- $\mathrm{GHz} \mathrm{H}_{2} \mathrm{O}$ maser components around IK Tau. The black outlines around coloured symbols show features detected by MERLIN in both 2000 and 2001.
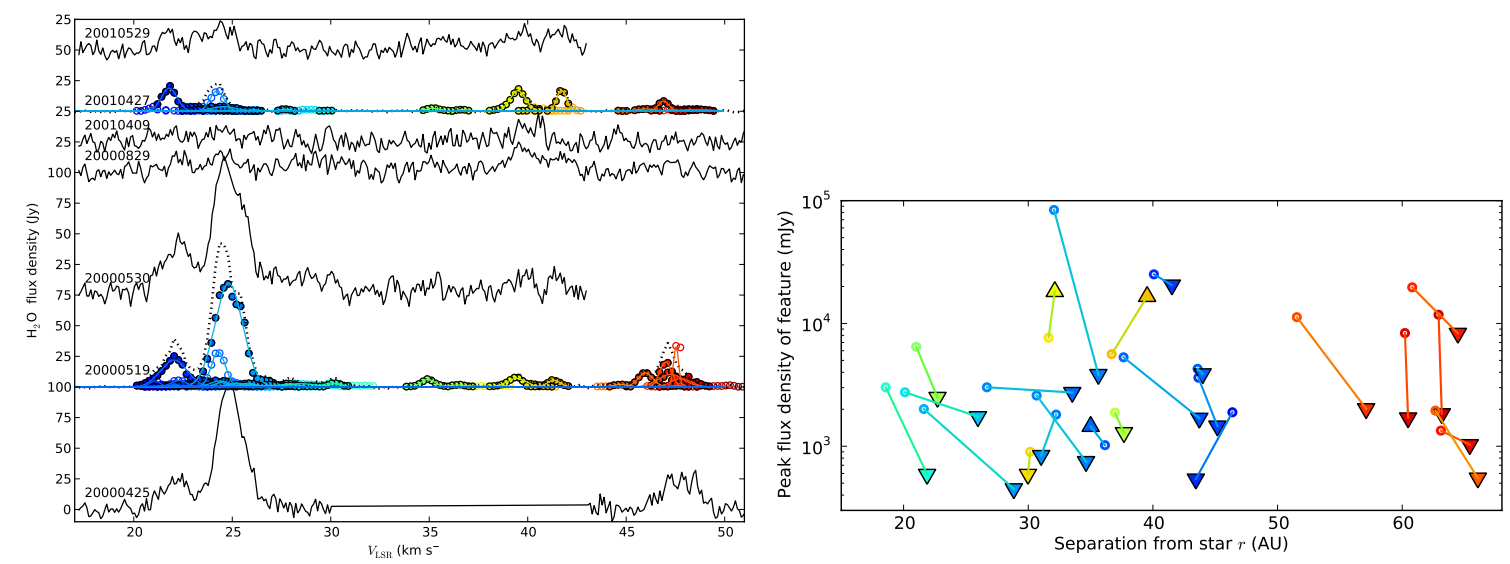

Figure 2: Spectra for the IK Tau MERLIN data shown in Fig. 1 and monotone Pushchino spectra taken at intervening epochs are shown on the left. The peak flux densities of the matched MERLIN features are shown as a function of radial separation from the star on the right, the triangles marking the later epoch.

with spatial features, fade and reappear on short timescales. This implies that the masers switch on and off (in our direction) due to their sensitivity to small changes in excitation conditions or local turbulence, but the parent clouds persist for at least as long as the maser shell crossing time.

We assumed spherical symmetry to solve for the 3D velocity-position field of the shells. Fig. 2, right, shows that, for IK Tau, all but two matched maser features fall in brightness between epochs regardless of their distance from the star. All the stars in our sample show flux density changes which are either seemingly random with respect to distance from the star, or which occur in the same sense at any distance, even in RT Vir which was observed 6 times in ten weeks. This shows that most of the maser variability must be radiative in origin, since shocks could not travel the many-au depth of the shell in even a few years at the speeds required to retain molecules. There is some evidence for shocks in the stars with the deepest pulsations, probably at the inner edge of the shell [19]. Individual features can be amplified due to cloud overlap, as described (in AGN 'tori') 

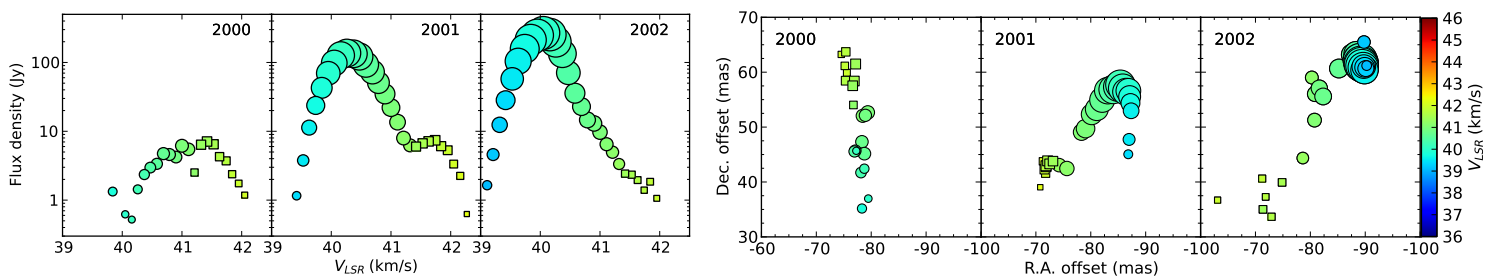

Figure 3: Spectra and positions of components making up two maser features in W Hya, circles and squares.

by [12] and we saw an instance of this in W Hya. Pushchino monitoring revealed a single peak had a flux density of a few hundred Jy prior to 2000-04-25 but had flared to $2800 \mathrm{Jy}$ on 2001-10-23, declining again by 2002-04-22. MERLIN caught the quiescent features in 1999 and 2000, and the start and finish of the flare [20]. Fig. 3 shows that the more blue-shifted feature appears to move from $\mathrm{S}$ to $\mathrm{N}$, passing in front of the other feature and brightening tenfold in the process.

Fig. 1 (right) shows that the emission has a well-defined inner rim $r_{\mathrm{i}}$ (as in all our sample). 22- $\mathrm{GHz} \mathrm{H}_{2} \mathrm{O}$ maser emission becomes possible where the density falls below the point at which collisions exceed the maser emission rate. The quenching number density is $\sim 5 \times 10^{15} \mathrm{~m}^{-3}$ at 1000 $\mathrm{K}$. However, the average number density at $r_{\mathrm{i}}$, estimated from species like $\mathrm{CO}$, is only a few $10^{14}$ $\mathrm{m}^{-3}$. This shows that the $22-\mathrm{GHz}$ emission traces clouds which are on average $\geq 45 \times$ the average wind density although they have a filling factor $<1 \%$. Between $20 \%$ and almost all the mass lost is concentrated in these clouds and 2-6 are formed per stellar cycle.

We found that the $\mathrm{H}_{2} \mathrm{O}$ maser cloud radii, of $0.6-9$ au, are closely proportional to $R_{\star}$. The RSG clouds are $\sim 10 \times$ the size of AGB clouds. This implies that the clouds are formed, and their properties are determined, at the surface of the star (not by effects such as cooling at larger distances from the star). Assuming radial expansion, their birth sizes are $5-10 \% R_{\star}$, similar to the size of convection cells in AGB stars and RSG modelled by e.g. [7] and [4].

\section{Resolving Betelgeuse}

Betelgeuse is the closest RSG, at $197 \mathrm{pc}$ [9], and hence has the largest angular $R_{\star}, 22.5$ mas at $2.2 \mu \mathrm{m}$ [18]. It is relatively young (M21ab) and masers have not been detected in its wind but the stellar radio continuum has been well-resolved by e-MERLIN [21] in July 2012 using $~ 400 \mathrm{MHz}$ effective bandwidth between 5.5-6.0 GHz. We used two imaging schemes, one with a tapered beam to optimise sensitivity and the other with partially uniform weighting to optimise resolution. The rms noises $\sigma_{\text {rms }}$ were 27 and $9 \mu \mathrm{Jy}_{\text {beam }}{ }^{-1}$, respectively. Fig. 4 (left) shows that, for the first time, we have resolved details of the stellar surface at high significance, revealing two hot-spots, 90 mas apart, with brightness temperatures $T_{\mathrm{B}}$ of $5400 \pm 600$ and $3800 \pm 500 \mathrm{~K}$. A few hotspots were detected previously with the less sensitive old MERLIN (right), requiring $T_{\mathrm{B}} \geq 6000 \mathrm{~K}$. The stellar disc has an average $T_{\mathrm{B}}$ of $1250 \pm 150 \mathrm{~K}$, consistent with earlier VLA images e.g. [16]. Harper \& Brown ([8]) compared HST UV data with radio measurements to show that the hottest stellar layers, at $\sim 1.45 R_{\star}$, are a mixture of photospheric and chromospheric emission. Although the chromosphere could reach $8000 \mathrm{~K}$, the filling factor is very small. Hence, the hot spots revealed at high resolution at $6 \mathrm{GHz}$ could be chromospheric patches, although a magnetic heating mechanism 

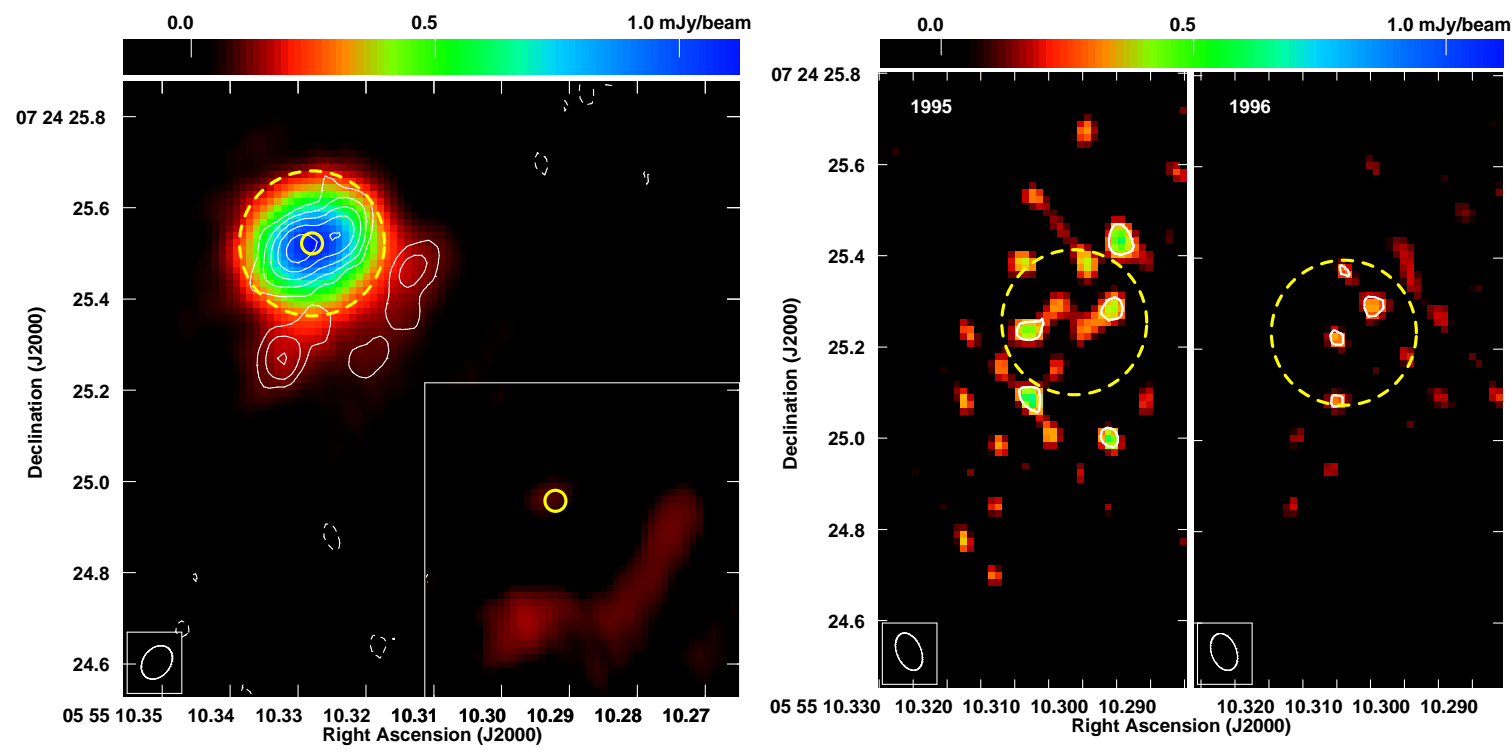

Figure 4: The colour image (left) shows Betelgeuse imaged in 2012 at 180-mas resolution, around 5.75 GHz. The white contours, at $(-1,1,2,4,8,16) \times 27 \mu \mathrm{Jy}_{\text {beam }}{ }^{-1}$, show the star at $60 \times 80$ mas resolution. The insert shows the lower-resolution image after subtracting the central star. The solid and dashed yellow circles represent the photosphere and the radius of 5-GHz emission measured by [16], respectively. The right-hand images show 1995 and 1996 images, contours at 600 and $400 \mathrm{mJy}^{\text {beam }}{ }^{-1}\left(4 \sigma_{\mathrm{rms}}\right)$, respectively.

is likely to be required to maintain high temperatures at the $6 \mathrm{GHz}$ radius, $5 R_{\star}$. Other possibilities include convective levitation of hotter, inner gas, abnormally optically thin windows allowing hotter layers to shine through, shock heating by pulsations, or some combination of these factors.

The SW extension (Fig. 4, left and insert) has a mass tentatively estimated at $\sim 1.5 \times 10^{-6} \mathrm{M}_{\odot}$. It is in a similar direction to an IR plume imaged by [13] and a CO clump detected by [17], although the distances and timescales suggest that these are the products of separate ejection events. Clumps were detected at a range of position angles by [14]. These phenomena are broadly consistent with the clumpy mass loss seen in masers and other molecules, and with a link between mass ejection and the larger-scale convection cells modelled by [5] on the basis of VLTI observations [10].

\section{Summary and future work}

We have used 22- $\mathrm{GHz} \mathrm{H}_{2} \mathrm{O}$ maser observations at sub-au resolution to investigate mass loss from cool, evolved stars. Comparison between multi-epoch MERLIN imaging and Pushchino monitoring shows that the clouds are likely to survive at least the maser shell crossing time (decades or centuries) but the masers wax and wane with local conditions. Masers throughout the shell, tens or more au thick, often vary together on timescales of a year or faster, which must be due to radiative processes. Individual maser features may flare due to cloud overlap along the line of sight, as seen in W Hya. The maser clouds are typically $\geq 30 \times$ the average wind density, but with a filling factor $<1 \%$. The cloud radius is proportional to the stellar radius, consistent with models which suggest a connection between mass loss mechanisms and stellar convective cells on scales around $0.1 R_{\star}$. e-MERLIN observations of Betelgeuse at 5.6-6 GHz resolved a disc of average size and brightness 
temperature consistent with earlier VLA measurements, showing that the bulk of the 6-GHz atmosphere of Betelgeuse is at $\sim 1250 \mathrm{~K}$. Hot spots at $\sim 4000-6000 \mathrm{~K}$ (also seen, at low significance, in old MERLIN images) are consistent with the presence of chromospheric patches with a low filling factor, although optical depth effects, convection or pulsation could also be involved.

Results from MERLIN, the EVN and Nançay will reveal whether $\mathrm{OH}$ mainline masers trace lower-density gas interleaving the clumps. ALMA observations of higher-frequency $\mathrm{H}_{2} \mathrm{O}$ masers, simultaneously detecting the star and dust, will reveal the link between dust formation and mass loss (see also Laing, these proceedings). Multi-frequency ALMA, VLA and e-MERLIN observations of stellar continuum will trace layers which are cooler and thus are optically thick to greater radii, with increasing frequency. This will show whether disturbances are correlated across the disc, suggesting pulsation, or on smaller scales, consistent with convection. Linked VLBI and ALMA $\mathrm{SiO}$ maser imaging will show the relationship between stellar surface events and mass loss.

\section{References}

[1] Assaf, K. A., Diamond, P. J., Richards, A. M. S. \& Gray, M. D., 2011, MNRAS, 415, 1083

[2] Bowen, G. H., 1988, ApJ, 329, 299

[3] Chapman, J. M. \& Cohen, R. J., 1986, MNRAS, 220, 513

[4] Chiavassa, A., Plez, B., Josselin, E. \& Freytag, B., 2009, A\&A, 506, 1351

[5] Chiavassa, A. et al., 2010, A\&A, 511, A51

[6] Danchi, W. C., Bester, M., Degiacomi, C. G., Greenhill, L. J. \& Townes, C. H., 1994, AJ, 107, 1469

[7] Freytag, B. \& Höfner, S., 2008, A\&A, 483, 571

[8] Harper, G. M. \& Brown, A., 2006, 646, 1179

[9] Harper, G. M., Brown, A. \& Guinan, E. F., 2008, AJ, 135, 1430

[10] Haubois, X. et al., 2009, A\&A, 508, 923

[11] Imai, H. et al., 2003, ApJ, 590, 460

[12] Kartje, J. F. Königl, A. \& Elitzur, M., 1999, ApJ, 513, 180

[13] Kervella, P. et al., 2009, A\&A, 504, 115

[14] Kervella, P. et al., 2011, A\&A, 531, A117

[15] Le Bertre, T., Matthews, L. D., Gérard, E. \& Libert, Y., 2012, MNRAS, 422, 3433

[16] Lim, J., Carilli, C. L., White, S. M., Beasley, A. J., \& Marson, R. G. 1998, Nat, 392, 575

[17] O'Gorman, E. et al., 2012, AJ, 144, 36

[18] Perrin, G. et al., 2004, A\&A, 418, 675

[19] Richards, A. M. S., Elitzur, M., \& Yates, J. A. 2011, A\&A, 525, 56

[20] Richards, A. M. S. et al., 2012, A\&A, 546, 16

[21] Richards, A. M. S. et al., 2013, MNRAS, submitted

[22] Wittkowski, M., Boboltz, D. A., Ohnaka, K., Driebe, T., \& Scholz, M. 2007, A\&A, 470, 191

[23] Woitke, P. 2006, A\&A, 460, L9

[24] Yates, J. A., \& Cohen, R. J. 1994, MNRAS, 270, 958 